\title{
Le Modèle dynamique de changement en soutien à l'accompagnement: vers des pratiques plus inclusives
}

Nadia Rousseau

Université du Québec à Trois-Rivières (Canada)
Dynamic Model of Change in School for more inclusive practices

\section{ésumé}

Le développement de pratiques plus inclusives repose sur plusieurs injonctions internationales centrées sur le droit à une éducation offerte dans un cadre intégré, à l'intérieur d'un seul et même système d'éducation public et inclusif. Toutefois, les pratiques dites plus inclusives reposent sur une culture de changement dans l'école. C'est en réponse à la nécessité de soutenir ce changement que huit conseils scolaires ontariens se sont approprié le Modèle dynamique de changement accompagné en contexte scolaire à travers un projet d'accompagnement de trois ans. Cet article présente ce projet, les composantes du Modèle dynamique de changement accompagné en contexte scolaire, ainsi que les retombées du projet et les défis vécus par les accompagnateurs.

Mots-clés

Inclusion scolaire, pratiques inclusives, changement de pratique, Modèle dynamique de changement, accompagnement au changement.

Abstract

The development of more inclusive practices is based on international injunctions focusing on equal access and the right to an education offered within a single system of public and private education. However, more inclusive practices are based, among other things, on a culture of change within the school. In response to the need to support change at school, eight Ontario school boards have adopted the Dynamic Model of Change in School through a three-year supported project. This article presents this project, the components of the Model exploited in the project, the contributions of the project as well as the challenges experienced by the participants.

\section{Keywords}

Inclusive education, inclusive practices, practice change, Dynamic Model of Guided Change, support for change.

\section{Les injonctions internationales et les conventions locales}

Selon Ramel et Vienneau (2016), l'inclusion scolaire « repose [...] sur des bases sociologiques qui prennent forme dans un mouvement de revendication des droits civiques et s'ancrent en particulier dans l'exigence formulée par les milieux associatifs de droits éducatifs pour les élèves en situation de handicap ou en très grande difficulté »(p.25). En d'autres mots, l'inclusion cherche à contrecarrer les processus d'exclusion qui se vivent à l'école et à affirmer l'éducation pour tous dans son projet éducatif(Prud'homme et Ramel, 2016). Déjà, les années 1959 à 1989 sont marquées par une série d'injonctions internationales ayant comme thématique centrale les droits de l'enfant, notamment le droit à une intégration sociale pleine et entière, à une éducation et à des soins spécialisés (Ramel et Vienneau, 2016). À titre d'exemple, le Programme d'action mondial concernant les personnes handicapées de l'ONU (1982) stipule : «Dans toute la mesure du possible, l'enseignement des personnes handicapées devrait se faire dans le cadre du système général d'enseignement» (p. 28). Les injonctions internationales produites dans les années 1990 abordent plutôt un discours centré sur l'égalité d'accès et le droit à une éducation offerte dans un cadre intégré, à l'intérieur d'un seul et même système d'éducation public et inclusif (Ramel et Vienneau, 2016). À ce titre, la Déclaration de Salamanque et Cadre d'action pour l'éducation et les besoins spéciaux constitue une figure marquante (UNESCO, 1994). Quatre extraits de cette déclaration sont particulièrement significatifs, eu égard à la place légitime de la diversité des élèves qui composent l'école :

- "Chaque enfant a des caractéristiques, des intérêts, des aptitudes et des besoins d'apprentissage qui lui sont propres ». (p. viii) 
- «Les systèmes éducatifs doivent être conçus et les programmes appliqués de manière à tenir compte de cette grande diversité de caractéristiques et de besoins». (p. viii)

- «Les personnes ayant des besoins éducatifs spéciaux doivent pouvoir accéder aux écoles ordinaires, qui doivent les intégrer dans un système pédagogique centré sur l'enfant, capable de répondre à ces besoins ». (p. viii)

- «Les écoles ordinaires ayant cette orientation intégratrice constituent le moyen le plus efficace de combattre les attitudes discriminatoires, en créant des communautés accueillantes, en édifiant une société intégratrice et en atteignant l'objectif de l'éducation pour tous » (p. ix).

Enfin, les injonctions internationales des années 2000 abordent plus spécifiquement la question de l'inclusion scolaire et des transformations qu'elle sous-tend (Ramel et Vienneau, 2016). Le document Principes directeurs pour l'inclusion : Assurer l'accès à «l'Éducation pour tous »(UNESCO, 2005) constitue un cadre clé en la matière. En effet, ce texte, contrairement aux précédents, insiste sur l'importance de tenir compte de tous les apprenants, avec ou sans handicap. Qui plus est, il insiste sur le fait que l'éducation pour tous oblige une transformation de l'école actuelle. Cela dit, bien que plusieurs textes d'encadrement encouragent le développement de pratiques plus inclusives (par exemple, ONU, 1993; UNESCO, 1994), ils offrent peu de pistes relativement à l'actualisation de l'inclusion scolaire par les acteurs de l'éducation et rendent difficilement compte de la complexité de l'inclusion scolaire (Ramel et Vienneau, 2016). C'est là qu'entrent en jeu les textes d'encadrement locaux. Dans le cas de l'Ontario, L'apprentissage pour tous, Guide d'évaluation et d'enseignement efficaces pour tous les élèves de la maternelle à la $12^{e}$ année (Ministère de l'Éducation de l'Ontario [MEO], 2013) en est un exemple. En effet, ce guide :

décrit les approches pédagogiques fondées sur une des plus importantes conclusions des recherches en éducation depuis l'an 2000, soit que tous les élèves apprennent mieux lorsque l'enseignement, les ressources et le milieu d'apprentissage correspondent bien à leurs points forts, leurs intérêts, leurs besoins et leur niveau de préparation à l'apprentissage. (p. 7)

Le guide met également de l'avant une série de sept principes sous-jacents aux approches proposées, dont quatre qui donnent le ton aux actions prises à l'école pour soutenir le rendement et le bienêtre des élèves : "Tous les élèves peuvent réussir »; "les titulaires de classe sont les acteurs clés du développement des compétences des élèves en littératie et en numératie »; « le personnel enseignant a besoin de l'appui de la communauté pour créer un environnement d'apprentissage favorable à tous les élèves »; et « l'équité n'est pas synonyme d'uniformité » (p. 7). Bien que les textes d'encadrement locaux constituent une avancée importante en matière d'inclusion scolaire, ils permettent difficilement, à eux seuls, le développement de pratiques plus inclusives.

\section{Certains défis associés au développement de pratiques plus inclusives}

L'analyse des encadrements et des pratiques inclusives de sept provinces canadiennes, soit l'Alberta, le Manitoba, le Nouveau-Brunswick, la Nouvelle-Écosse, l'Ontario, le Québec et la Saskatchewan, met en évidence que le développement de telles pratiques est certes favorisé par des politiques provinciales explicites, mais que la seule présence de ces politiques est insuffisante pour assurer un changement de pratique qui répond à la complexité de l'inclusion scolaire (Rousseau, Borri-Anadon et St-Vincent,

6 - Formation et profession 28(1), 2020 
2014) ${ }^{1}$. Ce constat s'explique par le fait que les politiques facilitent la mise en place de conditions administratives liées à l'inclusion, sans pour autant aborder d'autres conditions telles que l'attitude des enseignants envers la diversité (AuCoin, Goguen et Vienneau, 2011). De plus, les défis associés à la compréhension même du concept d'inclusion, le manque de connaissances relatives aux stratégies de mise en œuvre de pratiques plus inclusives, voire des fondements éthiques, moraux et sociaux de l'inclusion, de même que la transformation des rôles que sa pratique entraîne sont autant d'éléments à prendre en considération. Précisons que l'inclusion scolaire s'appuie sur des principes d'égalité des droits éducatifs et d'accès universel à l'institution, qui « joue un rôle fondamental dans l'apprentissage des règles de la vie en société et dans le processus d'intégration sociale des individus » (Ducharme, 2008, p. 5). Rappelons également que l'inclusion scolaire reconnaît la légitimité de la diversité en éducation, quelle que soit l'étendue des différences susceptibles d'être rencontrées dans une classe (Prud'homme, Vienneau, Ramel et Rousseau, 2011; Vienneau, 2006). D'ailleurs, la prise en compte de la diversité des besoins des élèves est nécessaire pour maximiser la participation à l'apprentissage, à la vie sociale et culturelle de l'école et de la communauté (Booth et Ainscow, 2004). Les valeurs associées à l'éducation inclusive comprennent donc : le respect des différences et de l'unicité de chaque apprenant (légitimité de la diversité), la coopération et l'entraide (simplement pour aider, par altruisme, par bienveillance) et la reconnaissance qu'il y a une part de l'autre dans la construction de soi (l'altérité) (Vienneau, 2002, 2016). Cette façon de concevoir l'école oblige à une culture de changement en son sein (Bélanger et Duchesne, 2011b; Rousseau, Point, Vienneau, Desmarais et Desmarais, 2017), une transformation de l'organisation scolaire et des représentations que font les enseignants de la diversité (Dyson et Millward, 1997; Kahn, 2010), en plus d'entraîner un changement de paradigme important qui place l'école en situation d'adaptation devant la diversité des élèves, et non l'inverse (Bélanger et Duchesne, 2011a; Peters, 2007).

Considérant la place prépondérante qu'occupe la culture de changement en matière de développement de pratiques plus inclusives et les besoins qui découlent des politiques ontariennes en matière d'éducation inclusive, une recherche collaborative s'avère prometteuse pour soutenir l'appropriation du Modèle dynamique de changement accompagné en contexte scolaire pour le bien-être et la réussite de tous (Rousseau, 2012) $)^{2}$ par les personnes responsables d'accompagner les équipes-écoles de leurs propres conseils scolaires en matière d'inclusion. Fidèle à l'esprit de la recherche collaborative, la démarche de recherche ne vise pas la validation du Modèle, mais bien la formation professionnelle des participants à travers son appropriation. Il apparaît toutefois judicieux de préciser que ce Modèle de changement accompagné est induit de données de recherche portant sur la mise en œuvre d'un processus de changement visant la réussite d'une diversité d'élèves en contexte québécois. Comme en témoigne Baby (2012), il constitue un « outil de développement professionnel modifiant les pratiques pédagogiques chez les enseignants qui souhaitent prioriser la réussite et le bien-être de tous les élèves » (p. 218). Ainsi, il se distingue d'autres modèles plus généraux par sa posture plus spécifique et contextualisée. Le Modèle, à la lumière de certains constats issus de l'étude du changement et des fonctions d'accompagnement au changement, repose sur une démarche d'accompagnement qui gravite autour de trois phases du changement planifié, soit la décristallisation, l'instauration du changement et la recristallisation (Schermerhon, Hunt et Osborn, 2002). La première phase (décristallisation) est une étape préliminaire visant la remise en question des attitudes et des comportements présents contribuant à faire émerger clairement le besoin de changement. La deuxième phase (instauration du changement) 
consiste à implanter des mesures visant à changer une situation donnée par la modification d'une variété de paramètres comme les tâches, la structure, la technologie. Enfin, la troisième et dernière phase (recristallisation) vise à consolider et assimiler à long terme les acquis du changement. Ces phases répondent à la prise en compte de la complexité du changement en contexte scolaire. À ce titre, une synthèse d'écrits réalisée par Fournier et Fréchette (2012) met clairement en évidence que les principaux acteurs que sont les enseignants et les éducateurs ont avantage à participer aux processus décisionnels entourant le changement dans leur milieu. Qui plus est, les fonctions d'accompagnement de Garant (2003) ont également contribué au développement du Modèle, notamment la fonction de catalyseur, où l'accompagnateur cherche à provoquer un conflit sociocognitif pour aider les acteurs à réfléchir toujours plus; la fonction de facilitateur, où l'accompagnateur soutient la réflexion sur la juste perception des actions posées à l'école, et ce, dans un climat sécuritaire; la fonction de conseil, où l'accompagnateur adopte une posture de médiation et de compagnonnage réflexif; et enfin, la fonction de liaison, où l'accompagnateur contribue à la création de liens avec le monde extérieur tant en matière de connaissances issues de la recherche que de ressources humaines susceptibles de contribuer au projet de changement.

\section{La recherche collaborative}

La recherche collaborative s'inscrit dans « une volonté de formation des individus par une démarche réflexive qui va s'adjoindre d'une intention de recherche» (Bourassa et Boudjaoui, 2012, p. 5). Ainsi, les participants ne sont pas objets de recherche, mais bien acteurs principaux de leur projet de développement professionnel. Dans le cas qui nous préoccupe, les participants, au nombre de 21, sont tantôt conseillers pédagogiques, coordonnateurs des services aux élèves ayant des besoins particuliers ou directeurs d'établissement issus de huit conseils scolaires francophones de l'Ontario. Ces participants ont tous pour mandat de soutenir le développement de pratiques plus inclusives dans les écoles de leurs conseils scolaires respectifs à travers un processus d'accompagnement. C'est dans ce cadre que ces derniers ont approché la chercheuse pour s'approprier le Modèle dynamique de changement accompagné dans ce contexte bien précis.

Le Modèle dynamique de changement accompagné offre un cadre de développement professionnel aux milieux scolaires désireux d'amorcer un projet de changement dans leur institution. Il vise le développement professionnel de l'ensemble des membres qui composent l'équipe-école pour ainsi arriver à une plus grande cohésion d'actions à travers une démarche longitudinale en réponse à un objectif commun d'amélioration de pratiques. La démarche entreprise, d'une durée de trois ans, s'appuie sur les trois phases du Modèle. Dès lors, les 21 participants se prêtent au processus de changement où la chercheuse joue le rôle d'animatrice ${ }^{3}$ (première étape de l'appropriation du Modèle par les participants). Ensuite, ces derniers accompagnent leurs propres milieux scolaires où ils deviennent eux-mêmes accompagnateurs au changement (deuxième étape de l'appropriation du Modèle par les participants). Cinq rencontres annuelles d'une journée (total de 15 rencontres sur 3 ans) réunissant les participants et la chercheuse permettent d'analyser en profondeur le Modèle et le rôle d'accompagnateur s'y rapportant. Ces rencontres permettent également de mettre en lumière à la fois les retombées du projet sur les participants et les milieux accompagnés, tout en portant une attention particulière aux défis rencontrés par les participants dans leur rôle d'accompagnateurs. 
Les outils de collecte de données mobilisés lors de ces 15 rencontres comprennent : le journal de bord de la chercheuse, où les questionnements, les impressions, voire les étonnements sont notés; la prise de notes, où l'ensemble des propos des participants est fidèlement consigné, et où l'interprétation de ces propos est ensuite validée avec les participants; les artefacts, où l'ensemble des productions formelles et informelles des participants est sujet à un entretien d'explicitation collectif visant à mettre en mots l'implicite derrière la production (Vermersch, 1996). Enfin, la rencontre-bilan (la quinzième) s'amorce par une réflexion individuelle sur l'expérience des participants. Cette réflexion individuelle constitue l'amorce de la synthèse collective. Comme en témoigne ce qui précède, il s'agit d'un processus de collecte et d'analyse inductive continu qui se fait avec les participants, soit une validation écologique des résultats avec les personnes concernées. Dès lors, l'ensemble des données validées par les participants, au fil des rencontres, a fait l'objet d'une codification suivant la méthode du codage par réseau (Cohen, Manion et Morrison, 2008) avec l'assistance du logiciel Atlas.ti. Ce codage inductif fonde la construction d'un système complexe de catégories et de sous-catégories qui, tout en permettant le classement de données qualitatives, assure la visibilité des liens qui les unissent. Le logiciel Atlas.ti constitue un outil clé dans ce type d'analyse (Weitzman, 2000). Cela dit, le logiciel ne supplante pas le processus de réflexion entourant le codage. Une fois la codification complétée, les codes induits ont permis l'élaboration d'une première catégorisation des résultats. Le même exercice a ensuite donné lieu à la thématisation des propos des participants.

Avant d'explorer les retombées et les défis associés à ce projet, il importe de présenter, même succinctement, les principales composantes du Modèle, qui rappelons-le, est construit autour de trois phases. L'annexe 1 offre une représentation visuelle du Modèle.

\section{Phase 1 - an 1 : La préparation au changement : trois étapes indissociables et inter- dépendantes}

Cette phase correspond à ce que Schermerhorn, Hunt et Osborn (2002) nomment la décristallisation. Elle comprend trois étapes :

\section{1) L'identification des défis perçus}

Cette étape oblige une réflexion visant l'identification des perceptions et, conséquemment, l'identification des croyances et des attitudes des membres qui composent le groupe. Les croyances renvoient à : « l'attitude intellectuelle d'une personne qui tient pour vrai un énoncé ou un fait sans qu'il y ait nécessairement une démonstration objective et acceptable de son attitude » (Bloch et al., 2002, cités dans Morin et Aubé, 2007, p. 60). Comme l'expliquent les auteurs, les croyances donnent lieu à des attitudes qui se traduisent par des réactions positives ou négatives face à une situation donnée, et ce, en fonction des sentiments ou des idées que provoque cette situation. Il s'agit là d'une étape « difficile », puisqu'elle incite les participants à se mettre à nu. À cette étape, les croyances partagées, les attitudes qu'elles génèrent et les discussions qui en émergent permettent la création d'un espace d'intersubjectivité où tous sont invités à prendre conscience individuellement et collectivement de ce qui, inévitablement, colore leurs perceptions du problème. 


\section{2) L'élaboration de la vision}

Cette étape renvoie à une réflexion favorisant la reconnaissance des erreurs de perception, le développement d'une compréhension empathique des élèves qui composent les classes et l'école, et la prise de conscience du potentiel individuel et collectif perçu qui habite les murs de l'école (tant pour les membres de l'équipe-école que pour les élèves). La réflexion entourant les erreurs de perception et la compréhension empathique facilite l'élaboration d'une vision commune que se donne l'école dans son projet de changement, vision qui tend à mobiliser le potentiel individuel et collectif de tous les acteurs de l'école. Cette étape en est vraiment une de coconstruction de sens qui oblige le dialogue, la prise de conscience de soi et des autres (collègues et élèves).

\section{3) L'articulation de la mission}

Cette étape met à profit tant la réflexion que les savoirs d'expérience, pour ainsi contribuer à l'identification d'objectifs communs et d'une compréhension commune de la visée de ces objectifs. Cette étape (tout comme la précédente) repose sur un état de conscience de ce qui est maintenant et de ce qui pourrait être. À cette étape, il faut parfois « retenir les troupes ", car certains aspirent à mettre de côté la réflexion et les prises de conscience quelque peu difficiles pour sauter, à pieds joints, dans l'action! Même dans un contexte de communication favorable, l'arrivée prochaine du passage à l'action crée une forme de fébrilité. Cela dit, cette troisième étape, bien que brève, contribue au développement d'une cohésion au sein de l'équipe-école (projet d'école plutôt que projet individuel).

\section{Phase 2 - an 2 : La réalisation du changement : deux étapes complémentaires}

Cette phase correspond à ce que Schermerhorn et al. (2002) nomment l'instauration du changement. Elle comprend deux étapes :

\section{1) La planification des actions}

À cette étape, les participants sont invités à réfléchir sur les actions qui pourraient le mieux contribuer à la réalisation de leur projet commun et du même coup, à évaluer la pertinence et l'efficacité connues des actions envisagées. Cette étape de validation de l'efficacité des actions anticipées résulte à la fois des savoirs d'expérience (équipe-école) et des savoirs scientifiques (équipe d'accompagnateurs). Ce qui retient l'attention durant cette première étape, ce sont les apprentissages qui s'y construisent par l'équipe-école. D'une part, il y a l'intégration des nouvelles connaissances par l'équipe-école, ce que Morin et Aubé (2007) nomment « apprendre que ». D'autre part, cette étape favorise aussi l'acquisition de savoir-faire et le développement de compétences, ce que Morin et Aubé (2007) nomment « apprendre à ». Selon Vienneau (2011), en contexte éducatif, le savoir-faire « correspond aux connaissances procédurales et conditionnelles» (p. 303). D'ailleurs, c'est ainsi que les participants transigent de la planification vers le champ expérimental du processus de changement, et ce, dans la plupart des cas, dans un contexte de collaboration et d'entraide. 


\section{2) La mise en ouvre des actions}

Cette deuxième étape est caractérisée par le passage à l'action. C'est à cette étape que les membres de l'équipe-école s'inscrivent dans la troisième dimension de l'acte d'apprendre, soit « apprendre » (Morin et Aubé, 2007). Comme l'expriment si bien les auteurs : «Apprendre c'est changer; c'est acquérir des connaissances et des compétences; c'est modifier ses attitudes et ses comportements pour être plus efficace qu'avant; c'est changer ses routines, ses habitudes et ses automatismes; c'est s'adapter à une nouvelle situation » (p. 195).

\section{Phase 3 - an 3 : L'intégration du changement : bilan et amorce de nouveaux défis}

La troisième et dernière phase du changement en contexte scolaire correspond à ce que Schermerhorn et al. (2002) nomment la recristallisation. Elle comprend une seule étape :

\section{1) Le bilan du changement}

L'étape du bilan traduit la fin d'un long parcours ou encore l'amorce de nouveaux défis. Cette étape repose à la fois sur un exercice de réflexion portant sur tout le chemin parcouru de même que sur l'efficacité des actions déployées. Dès lors, il y a vérification de l'atteinte des objectifs, et ce, tant sur le plan individuel que sur le plan collectif. Relativement aux objectifs personnels ou professionnels, il s'agit d'un moment fort qui mène vers l'évaluation de l'efficacité des nouvelles actions déployées et des apprentissages réalisés. Cette évaluation peut mener à une révision des actions prioritaires ou à un réajustement de certaines actions.

\section{Retombées du projet - constats de l'exercice du bilan}

D'entrée de jeu, précisons que la structure d'accompagnement des participants et les quinze rencontres entre participants et la chercheuse ont permis de créer un contexte de développement professionnel qui se rapproche du codéveloppement professionnel, comme explicité par Vandercleyen (2019). Il s'agit d'un regroupement qui mise sur le groupe et sur les interactions entre les participants pour favoriser une amélioration de leur pratique professionnelle à partir d'une démarche structurée de réflexion sur l'action où les personnes sont liées par des expertises partagées ou complémentaires et mobilisées par un projet commun; dans ce cas-ci, un projet de développement professionnel sur l'accompagnement au changement en soutien aux pratiques inclusives dans les écoles de l'Ontario. Riches de trois années d'échanges et de réflexions au sein de ce groupe, les participants sont arrivés à la construction de leur propre bilan à l'issue du projet (fidèles au Modèle, les participants ont complété la phase 3 du Modèle). Ce bilan gravite autour de trois postures : bilan propre aux participants dans leur rôle d'accompagnateurs au changement, bilan propre aux écoles accompagnées par ces derniers, et bilan relatif aux défis qu'engendre la posture d'accompagnateur au changement. 


\section{Bilan propre aux participants dans leur rôle d'accompagnateurs au changement}

Les participants confirment avoir fait de nouveaux apprentissages à travers leur expérience d'accompagnateurs au changement. D'une part, et comme en témoignent leurs propos, ils précisent avoir eu à s'approprier "diverses applications en soutien à l'enseignement et à l'apprentissage » de même que bon nombre de logiciels d'aide technologique destinés aux jeunes qui éprouvent une variété de difficultés (apprentissage, communication, organisation, mathématiques). D’autre part, ils estiment aussi que leur "bagage de connaissances" s'est largement bonifié en raison "de nombreuses lectures professionnelles réalisées pour soutenir le projet de changement des écoles» accompagnées. Les participants précisent que ces lectures étaient nécessaires dans leur rôle d'accompagnateurs afin de soutenir la réflexion des membres de l'équipe-école et accompagner ces derniers dans la prise de décisions relatives aux meilleures actions à privilégier pour réaliser leur objectif de changement de pratique. À ce titre, précisons que les thématiques de lecture pouvaient varier d'un accompagnateur à l'autre, en cohérence avec les projets de changement accompagnés.

Les participants estiment également que le projet d'accompagnement vers des pratiques plus inclusives est associé à "l'amélioration des compétences communicationnelles en accompagnement ». À ce titre, ils s'expriment particulièrement sur l'écoute active et sur l'écoute des besoins du personnel scolaire inhérente au processus d'accompagnement. Qui plus est, «le développement d'un esprit d'analyse » est également associé au projet. Plus spécifiquement, les participants estiment que pour accompagner le changement suivant le Modèle, l'accompagnateur doit nécessairement se doter d' "orientations claires ", se "fixer des objectifs précis", déterminer "des indicateurs de réussite afin de mieux évaluer le cheminement parcouru ». Enfin, le projet d'accompagnement aura également permis le « réseautage » entre plusieurs conseils scolaires de l'Ontario, qui, malgré leurs spécificités, partagent des réalités similaires (mêmes politiques scolaires, milieu francophone minoritaire, attitudes tantôt ouvertes tantôt plus réfractaires face à la diversité ou face aux technologies, etc.).

\section{Bilan propre aux écoles accompagnées par les participants-accompagnateurs}

Le bilan des participants est également riche de constats sur la portée de l'accompagnement sur le changement de pratiques des milieux accompagnés. Plus spécifiquement, les participants estiment que le personnel scolaire des milieux accompagnés a développé des pratiques réflexives plus poussées. À ce titre, le questionnement relatif à l'efficacité (ou non) des interventions usuelles et de celles mises en place retient l'attention. Ce serait d'ailleurs ce questionnement qui permettrait au personnel scolaire d'amorcer le changement de certaines pratiques pédagogiques. Le développement de pratiques réflexives plus efficaces serait aussi le fruit de l'identification d'un leader et de l'exercice du leadership par cette personne dans le projet de changement. Ainsi, ce leader, par ses questions et ses propres réflexions, contribuerait à maintenir un haut degré de réflexion pédagogique, indépendamment des rencontres formelles de travail sur le changement. Les participants identifient également le développement d'habitudes d' "échanges de pratiques pédagogiques gagnantes entre le personnel scolaire». Ces échanges de pratiques auraient principalement porté sur «la planification de l'enseignement qui tient compte de la différenciation et des technologies » de soutien à l'enseignement et à l'apprentissage. Cette planification, que les participants qualifient de "plus efficace ", aurait également permis de diminuer l'isolement des élèves ayant des difficultés d'apprentissage au sein de la classe. Les participants relèvent aussi une motivation 
accrue chez certains enseignants d'écoles accompagnées. Ce faisant, ces derniers auraient même créé des guides variés pour « diverses applications visant le développement de l'autonomie chez les collègues et les élèves ", eu égard aux applications et aides technologiques disponibles pour enseigner et pour apprendre. D'ailleurs, les participants estiment que les écoles accompagnées ont pu «se familiariser en contexte réel d'utilisation avec les technologies "; certains milieux ayant profité de leurs projets de changement pour faire l'achat d'outils technologiques additionnels. Tout comme pour les accompagnateurs, le personnel des écoles accompagnées aurait également bonifié leur bagage de connaissances par la réalisation de lectures professionnelles.

\section{Bilan relatif aux défis qu'engendre la posture d'accompagnateur au changement}

Le bilan des participants au projet aura également permis de mettre en évidence certains défis engendrés par la posture d'accompagnateur au changement. En effet, le fait d'assumer le rôle d'accompagnateur au changement d'un milieu scolaire n'a pas été perçu comme un geste professionnel anodin par les participants-accompagnateurs. Deux enjeux majeurs sont mis de l'avant. Le premier enjeu relève à la fois des capacités communicationnelles des accompagnateurs et des capacités mobilisées pour susciter l'engagement du personnel scolaire autour d'un projet commun en réponse à des orientations ministérielles. Dès lors, "développer des stratégies pour convaincre et motiver les gens à s'engager dans un projet de changement» constitue un réel défi. D'une part, les participants-accompagnateurs estiment qu'il faut "respecter le cheminement professionnel » du personnel scolaire concerné, "tout en poussant suffisamment pour les faire avancer (zone proximale) ». D'autre part, le degré de mobilisation du personnel peut « déstabiliser la planification» des actions de l'accompagnateur. C'est ici que l'écoute des besoins du personnel scolaire joue un rôle important, tout comme le bagage de connaissances de l'accompagnateur nécessaire pour favoriser une réflexion en profondeur sur certains enjeux éducatifs propres à chaque milieu accompagné. À ce titre, les participants-accompagnateurs disent «vivre avec des incertitudes » quant au niveau de connaissances qu'ils ont. Trois questions les habitent :

- Serons-nous en mesure de répondre aux questions du personnel scolaire?

- Avons-nous suffisamment de connaissances pour les appuyer?

- Pouvons-nous les guider?

Pour atténuer ces incertitudes, les participants-accompagnateurs ont recours aux lectures professionnelles, aux échanges en profondeur lors des rencontres collectives ou aux rencontres de travail/planification avec les professionnels de leurs propres conseils scolaires. Ce deuxième enjeu ouvre la porte à un défi organisationnel : le temps. Ainsi, les participants-accompagnateurs reconnaissent qu'accompagner le changement les oblige à trouver du temps "pour gérer le projet de changement et accompagner le personnel dans ce projet ».

\section{La portée de l'apprentissage collectif}

Le projet d'accompagnement au changement accompagné vers des pratiques plus inclusives, appuyé par le Modèle, met clairement en évidence la portée d'une approche fondée sur le groupe et la réflexion collective d'acteurs ayant des expertises partagées dans l'appropriation de leur rôle d'accompagnateur au changement. Cette approche s'inscrit dans une perspective socioconstructiviste de l'accompagnement 
qui «suppose le développement de compétences professionnelles pour l'accompagnement où les personnes accompagnatrices comprennent et s'approprient le changement jusqu'à l'approfondir afin de soutenir d'autres personnes dans cette démarche » (Lafortune, 2008, p. 11). Cette approche s'inscrit également dans l'esprit du groupe de codéveloppement professionnel réunissant des acteurs qui souhaitent s'entraider dans leur processus d'apprentissage. À ce titre, Vandercleyen (2019) précise :

Ces personnes sont liées par des expertises partagées ou complémentaires et mobilisées par un projet commun : devenir plus efficaces dans leur pratique professionnelle. Elles réfléchissent ensemble et échangent à partir de préoccupations professionnelles vécues, et où l'expérience de chacun devient une ressource mise à disposition de tous. Le moyen privilégié est une démarche structurée de consultation lors de laquelle, dans une perspective de recherche conjointe, les participants échangent afin d'enrichir leur compréhension et d'élargir leur pouvoir d'action. (p. 17)

Le bilan réalisé par les participants au projet met aussi de l'avant la nécessité, pour l'accompagnateur au changement, d'accueillir et d'écouter le personnel scolaire, constat similaire à ceux de Le Bouëdec, Du Crest, Pasquier et Stahl (2001), qui identifient l'accueil et l'écoute comme étant une fonction essentielle de l'accompagnement. Dans le même sens, Lafortune (2008) estime que l'accompagnateur au changement doit maîtriser une série de compétences, dont celle de " prendre en compte la dimension affective dans l'accompagnement d'un changement » (p.3). Cette compétence renvoie notamment à la :

[...] présence affective : tenir compte de la dimension affective auprès des personnes formées à l'accompagnement ou des personnes accompagnées [... le] modelage affectif : montrer qu'on tient compte de la dimension affective dans l'intervention auprès des personnes accompagnées [... et l'] instrumentation affective : donner des idées pour tenir compte de la dimension affective dans les actions ultérieures (p. 65).

D'ailleurs, pour les participants au projet d'accompagnement suivant le Modèle, la maîtrise de cette compétence est particulièrement importante dans la première phase du changement (la préparation au changement), où les réflexions entourant les croyances et les valeurs éducatives sont parties prenantes des échanges.

Dès lors, les compétences communicationnelles se dessinent comme une métacompétence fondamentale dans le processus d'accompagnement au changement. À ce titre, l'écoute, le questionnement et le feedback caractérisent les habiletés communicationnelles favorables non seulement à l'accompagnement, mais plus largement à la mobilisation des équipes accompagnées (Cormier, 2011). De plus, une communication qui vise à développer une relation et non pas seulement à transmettre l'information constitue une clé dans la mobilisation en vue d'un changement (Gagnon, 2012). La nécessité de développer une telle relation entre accompagnateur et accompagnés est également avancée par Lafortune (2008). Lafranchise et al. (2019) voient également à cette compétence communicationnelle la prise en compte de l'affectivité.

Chose certaine, l'appropriation d'un rôle d'accompagnateur au changement soulève des incertitudes qui tendent à s'estomper au fil du temps, notamment à travers l'exercice de l'accompagnement soutenu par un processus d'accompagnement et de réflexion collective en profondeur en lien à ce rôle. À ce 
titre, Vallerand (2006) et Lafortune (2008) reconnaissent également qu'accompagner le changement nécessite un certain degré de tolérance face à l'incertitude qu'engendrent l'instabilité, la recherche de sens et le tâtonnement inhérent à la réflexion collective. Qui plus est, le changement vers des pratiques plus inclusives est déjà, en soi, lié à l'incertitude chez les acteurs scolaires (Bélanger et Duchesne, 2011b; Rousseau, Point, Vienneau, Desmarais et Desmarais, 2017) et oblige la prise de conscience de ses propres cadres interprétatifs (LaBoskey, 2007) de même que la reconstruction de ses cadres par la confrontation de ces dernières avec des savoirs formalisés ou d'autres grilles de lecture (Saussez et Ewen, 2006; Wittorski, 2001). Toutefois, pour les participants à ce projet d'accompagnement, ces moments d'incertitudes ont su apporter certains bienfaits, dont le développement d'une meilleure connaissance de soi, en plus d'agir comme levier efficace dans le développement de nouvelles connaissances à travers les lectures professionnelles et le partage d'expertise.

\section{Conclusion}

Cet article rend compte d'un projet d'accompagnement visant, d'une part, à soutenir le développement professionnel d'accompagnateurs au changement de huit conseils scolaires francophones de l'Ontario par l'appropriation du Modèle dynamique de changement accompagné et, d'autre part, à soutenir les participants-accompagnateurs dans leurs propres accompagnements en contexte scolaire ayant pour objectif le développement de pratiques plus inclusives en réponse aux orientations ministérielles. L'approche d'accompagnement mise de l'avant, qui s'apparente au codéveloppement professionnel et à une perspective socioconstructiviste du changement, s'est révélée une pièce maîtresse du processus d'accompagnement, permettant aux participants de consolider un réseau d'expertise entre les conseils scolaires certes, mais également d'adopter une posture réflexive collective où les savoirs d'expérience occupent une place prépondérante dans l'appropriation de nouveaux savoirs. Les compétences communicationnelles semblent en outre jouer un rôle de premier plan dans le processus d'accompagnement vers des pratiques plus inclusives, notamment l'écoute active et l'écoute des besoins du personnel scolaire. Comme d'autres écrits l'auront stipulé, l'accompagnement au changement peut également générer certaines vulnérabilités qui, semble-t-il, s’atténuent au fil du temps, notamment par le développement de nouvelles connaissances (savoirs) et de nouvelles compétences (savoir-faire) dans un contexte où la réflexion en profondeur contribue au développement d'une meilleure connaissance de soi.

À la lumière des résultats de cette étude, l'appropriation des connaissances issues de la recherche dans l'exercice du rôle d'accompagnateur au changement auprès de ses pairs retient l'attention. En effet, comment soutenir cette appropriation alors que des travaux tendent à démontrer que le champ de l'éducation s'expose peu à ces connaissances en raison de leur caractère peu contextualisé, vulgarisé et synthétisé (voir par exemple, Dagenais, 2007; Janosz et al., 2010; Landry et al., 2008; Marion et Houlfort,2015; Ramdé,2012), contrairement à d'autres champs disciplinaires? De plus, le défi du temps dans un processus de changement accompagné en contexte scolaire continue d'être une préoccupation majeure (temps de concertation, temps de préparation, temps de lecture, temps d'expérimentation, etc.). Ce résultat soulève la question de l'identification de balises à l'organisation scolaire optimale qui permettraient des projets de changement accompagné à l'intérieur du temps scolaire. Cela dit, l'étude témoigne de la grande capacité des milieux éducatifs à s'engager dans un projet de changement pour 
soutenir le bien-être et la réussite des jeunes qu'ils accompagnent, et démontre la valeur de projets où le codéveloppement professionnel peut se vivre.

\section{Notes}

1 Pour une analyse des politiques scolaires de la Nouvelle-Écosse, le lecteur peut consulter AuCoin, Goguen et Vienneau (2011). Pour une analyse des politiques scolaires du Manitoba, le lecteur peut consulter Duchesne et AuCoin (2011).

2 Le Modèle est le fruit d'un travail de recherche de la Chaire de recherche Normand-Maurice (2007-2012) réunissant Antoine Baby, Claude Dugas, Michelle Dumont, Julie Myre-Bisaillon, Sylvie Ouellet, Luc Prud'homme, Nadia Rousseau et Ghislain Samson.

3 Ce rôle se caractérise par le soutien, la transmission de consignes et d'informations, le questionnement réflexif, la transmission de connaissances et le partage d'expériences pratiques, la rétroaction, la clarification, et la prise en compte de la dimension affective, soit des rôles similaires à ceux décrits par Lafranchise, Paquet, Gagné et Cadec (2019).

\section{Références}

AuCoin, A., Goguen, L. et Vienneau, R. (2011). Pas plus spécial que nécessaire : analyse des politiques scolaires de la Nouvelle-Écosse à l'égard de l'inclusion scolaire des élèves avec handicaps. Éducation et francophonie, 39(2), 23-49. http://dx.doi.org/10.7202/1007726ar

Baby, A. (2012). En conclusion. L'applicabilité du Modèle. Dans N. Rousseau (dir.), Modèle dynamique de changement accompagné en contexte scolaire : pour le bien-être et la réussite de tous (p. 213-222). Québec, QC : Presses de l'Université du Québec.

Bélanger, N. et Duchesne, H. (dir.) (2011a). Des écoles en mouvement. Inclusion d'élèves en situation de handicap ou éprouvant des difficultés à l'école. Ottawa, ON : Presses de l'Université d'Ottawa.

Bélanger, N. et Duchesne, H. (2011b). Introduction. Des écoles en mouvement. Inclusion d'élèves en situation de handicap ou éprouvant des difficultés à l'école. Dans N. Bélanger et H. Duchesne (dir.), Des écoles en mouvement. Inclusion d'élèves en situation de handicap ou éprouvant des difficultés à l'école (p. 1-16). Ottawa, ON : Presses de l'Université d'Ottawa.

Booth, T. et Ainscow, M. (2004). Index for inclusion: developing learning, participation and play in early years and childcare. Bristol : Centre for Studies in Inclusive Education (CSIE).

Bourassa, B. et Boudjaoui, M. (dir.) (2012). Des recherches collaboratives en sciences humaines et sociales (SHS) : enjeux, modalités et limites. Québec, QC : Presses de l'Université Laval.

Cohen, L., Manion, L. et Morrison, K. (2008). The methodology of educational research. Athènes : Metaichmio.

Cormier, S. (2011). La communication et la gestion (2éd.). Québec, QC : Presses de l'Université du Québec.

Dagenais, C. (2007). Examen des mécanismes en jeu dans la décision des intervenants scolaires d’utiliser les connaissances issues de la recherche dans leurs pratiques. Rapport de recherche intégral, Université de Montréal.

Ducharme, D. (2008). L'inclusion en classe ordinaire des élèves à besoins éducatifs particuliers. Montréal, QC : Éditions Marcel Didier.

Duchesne, H. et AuCoin, A. (2011). Évolution des lois, règlements et politiques en matière d'inclusion scolaire au Manitoba : une analyse critique des directives administratives. Éducation et francophonie, 39(2), 50-70. http://dx.doi. org/10.7202/1007727ar

Dyson, A. et Millward, A. (1997). The reform of special education or the transformation of mainstream schools? Dans S. J. Pijl, C. J. W. Meijer et S. Hegarty (dir.), Inclusive education. A global agenda (p. 51-67). New York, NY : Routledge.

Fournier, H. et Fréchette, S. (2012). Vers une prise en compte de la complexité du changement en contexte scolaire québécois : une synthèse des publications. Dans N. Rousseau (dir.), Modèle dynamique de changement accompagné en contexte scolaire: pour le bien-être et la réussite de tous (p. 47-65). Québec, QC : Presses de 1'Université du Québec. 
Gagnon, Y.-C. (2012). Réussir le changement. Mobiliser et soutenir le personnel. Québec, QC : Presses de l'Université du Québec.

Garant, M. (2003). Pilotage et accompagnement de l'innovation dans un établissement scolaire. Paris : Ministère de l'Éducation nationale, de la Recherche et de la Technologie.

Janosz, M., Bélanger, J., Dagenais, C., Bowen, F., Abrani, P., Cartier, S. C., .. Turcotte, L. (2010). Aller plus loin, ensemble. Synthèse du rapport final d'évaluation de la stratégie d'intervention Agir Autrement. Montréal, QC : GRES, Université de Montréal.

Kahn, S. (2010). Pédagogie différenciée. Bruxelles : De Boeck.

LaBoskey, V. K. (2007). The methodology of self-study and its theoretical underpinnings. Dans J. J. Loughran, M. L. Hamilton, V. K. LaBoskey et T. Russell (dir.), International handbook of self-study of teaching and teacher education practice: Tome 2 (p. 817-869). Dordrecht : Springer.

Lafortune, L. (2008). Compétences professionnelles pour l'accompagnement d’un changement. Un référentiel. Québec, QC : Presses de l'Université du Québec.

Lafranchise, N., Paquet, M., Gagné, M.-J. et Cadec, K. (2019). L'accompagnement des animateurs pour optimiser les groupes de codéveloppement professionnel. Dans F. Vandercleyen, M. LHostie et M.-J. Dumoulin (dir.), Le groupe de codéveloppement professionnel pour former à l'accompagnement des stagiaires. Conditions, enjeux et perspectives (p. 155-182). Québec, QC : Presses de l'Université du Québec.

Landry, R., Becheikh, N., Amara, N., Ziam, S., Idrissi, O. et Castonguay, Y. (2008). La recherche, comment s'y retrouver? Revue systématique des écrits sur le transfert des connaissances en éducation. Repéré à http://www.education.gouv.qc.ca/ fileadmin/site web/documents/PSG/statistiques info decisionnelle/LaRechercheCommentSYRetrouver.pdf

Le Bouëdec, G., Du Crest, A., Pasquier, L. et Stahl, R. (2001). L'accompagnement en éducation et formation. Un projet impossible? Montréal, QC : L'Harmattan.

Marion, C. et Houlfort, N. (2015). Transfert de connaissances issues de la recherche en éducation : situation globale, défis et perspectives. Nouveaux c@hiers de la recherche en éducation, 18(2), 56-89. http://dx.doi.org/10.7202/1036033ar

Ministère de l'Éducation de l'Ontario (MEO). (2013). L'apprentissage pour tous, Guide d'éraluation et d'enseignement efficaces pour tous les élèves de la maternelle à la $12^{e}$ année. Repéré à http://www.edu.gov.on.ca/fre/general/elemsec/speced/ LearningforAll2013Fr.pdf

Morin, E. M. et Aubé, C. (2007). Psychologie et management (2 $2^{\mathrm{e}}$ éd.). Montréal, QC : Chenelière Éducation.

Organisation des Nations Unies (ONU). (1982). Programme d'action mondial concernant les personnes handicapées. Paris : ONU.

Organisation des Nations Unies (ONU). (1993). Règles pour l'égalisation des chances des handicapés. Repéré à www.un.org/ esa/socdev/enable/dissrfro.htm

Peters, S. (2007). Inclusion as a strategy for achieving education for all. Dans L. Florian (dir.), The Sage handbook of special education (p. 116-130). Londres : Sage. http://dx.doi.org/10.4135/9781848607989.n10

Prud'homme, L. et Ramel, S. (2016). Introduction. Dans L. Prud'homme, H. Duchesne, P. Bonvin et R. Vienneau (dir.), L'inclusion scolaire : ses fondements, ses acteurs et ses pratiques (p. 15-17). Bruxelles : De Boeck.

Prud'homme, L., Vienneau, R., Ramel, S. et Rousseau, N. (2011). La légitimité de la diversité en éducation : réflexion sur l'inclusion. Éducation et francophonie, 39(2), 6-22. http://dx.doi.org/10.7202/1007725ar

Ramdé, J. (2012). Utilisation des connaissances issues de la recherche en éducation. Validation d'un questionnaire et proposition d'un modèle (Thèse de doctorat, Université de Montréal). Repéré à https://papyrus.bib.umontreal.ca/xmlui/ $\underline{\text { handle/1866/8876 }}$

Ramel, S. et Vienneau, R. (2016). Des fondements sociologiques de l'inclusion scolaire aux injonctions internationales. Dans L. Prud'homme, H. Duchesne, P. Bonvin et R. Vienneau (dir.), L'inclusion scolaire : ses fondements, ses acteurs et ses pratiques (p. 25-38). Bruxelles : De Boeck Supérieur.

Rousseau, N. (2012). Modèle dynamique de changement accompagné en contexte scolaire : pour le bien-être et la réussite de tous. Québec, QC : Presses de l'Université du Québec. 
Rousseau, N., Borri-Anadon, C. et St-Vincent, L.-A. (2014). Léducation inclusive au Canada : préoccupations communes et particularités provinciales. Dans L. Carlson-Berg (dir.), La francophonie canadienne dans toutes ses couleurs et le défi de l'inclusion scolaire (p. 35-62). Québec, QC : Presses de l'Université Laval.

Rousseau, N., Point, M., Vienneau, R., Desmarais, M.-É. et Desmarais, K. (2017). Les apports et les limites liés aux pratiques inclusives et la place de la collaboration dans ces pratiques : une métasynthèse. Revue suisse des sciences de l'éducation, 39(1), 21-40. Repéré à https://www.pedocs.de/volltexte/2018/16094/pdf/SZBW 20171 Rousseau et al Les apports et les limites.pdf

Saussez, F. et Ewen, N. (2006). La démarche d'investigation critique, pierre angulaire d'une formation à des compétences professionnelles? D'un cadre conceptuel à l'expérimentation de projets de formation. Les cabiers scientifiques de l'ACFAS, $108,45-67$.

Schermerhorn, J. R., Hunt, J. G. et Osborn, R. N. (2002). Comportement humain et organisation (2 éd.). Saint-Laurent, QC : Éditions du renouveau pédagogique.

UNESCO. (1994). Déclaration de Salamanque et Cadre d'action pour l'éducation et les besoins spéciaux. Repéré à https:// unesdoc.unesco.org/ark:/48223/pf0000098427 fre

UNESCO. (2005). Principes directeurs pour l'inclusion : Assurer l'accès à «l'Éducation pour tous ». Repéré à https://unesdoc. unesco.org/ark:/48223/pf0000140224 fre

Vallerand, R. (2006). Le temps de l'incertitude: du changement personnel au changement organisationnel. Québec, QC : Presses de l'Université du Québec.

Vandercleyen, F. (2019). Le groupe de codéveloppement professionnel comme approche de formation continue. Définition, principes et fonctionnement. Dans F. Vandercleyen, M. L'Hostie et M.-J. Dumoulin (dir.), Le groupe de codéveloppement professionnel pour former à l'accompagnement des stagiaires. Conditions, enjeux et perspectives (p. 13-30). Québec, QC : Presses de l'Université du Québec. http://dx.doi.org/10.2307/j.ctvjk2wc0.8

Vermersch, P. (1996). L'entretien d'explicitation en formation initiale et en formation continue. Paris : ESF.

Vienneau, R. (2002). Pédagogie de l'inclusion : fondements, définition, défis et perspectives. Éducation et francophonie, XXX(2), 257-286. Repéré à https://www.acelf.ca/c/revue/revuehtml/30-2/10-vienneau.html

Vienneau, R. (2006). De l'intégration scolaire à une véritable pédagogie de l'inclusion. Dans C. Dionne et N. Rousseau (dir.), Transformation des pratiques éducatives : la recherche sur l'inclusion scolaire (p. 7-32). Québec, QC : Presses de l'Université du Québec.

Vienneau, R. (2011). Apprentissage et enseignement : théories et pratiques (2 éd.). Montréal, QC : Gaëtan Morin Éditeur.

Vienneau, R. (2016). Les fondements de l'inclusion scolaire. Introduction. Dans L. Prud'homme, H. Duchesne, P. Bonvin et R. Vienneau (dir.), L'inclusion scolaire : ses fondements, ses acteurs et ses pratiques (p. 21-24). Bruxelles : De Boeck Supérieur.

Weitzman, E. A. (2000). Software and qualitative research. Dans N. K. Denzin et Y. S. Lincoln (dir.), Handbook of qualitative research ( $2^{\mathrm{e}}$ éd., p. 803-820). Thousand Oaks, CA : Sage.

Wittorski, R. (2001). Contribution de l'apprentissage expérientiel et de la science-action à la pratique professionnelle. Dans M.-P. Makiewicz (dir.), Praticien et chercheur - Parcours dans le champ social (p. 107-119). Paris : L'Harmattan. 


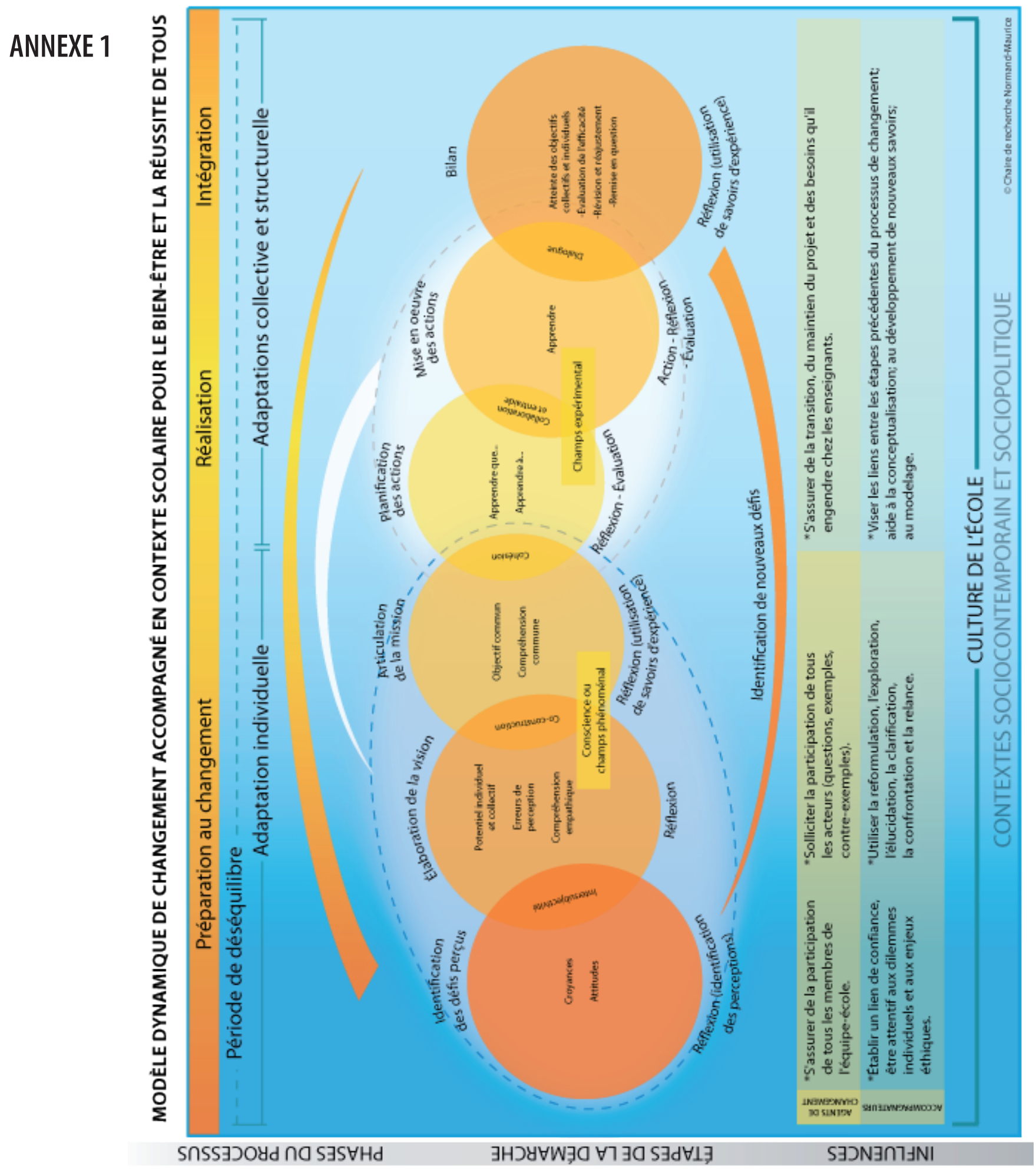

\section{Pour citer cet article}

Rousseau, N. (2020). Le modèle dynamique de changement en soutien à l'accompagnement : vers des pratiques plus inclusives. Formation et profession, 28(1), 5-19. http://dx.doi.org/10.18162/fp.2020.526 